CLINICAL STUDY

\title{
Oxidised low-density lipoprotein concentration - early marker of an altered lipid metabolism in young women with PCOS
}

\author{
Djuro Macut, Svetozar Damjanović, Dimitrios Panidis ${ }^{1}$, Nikolaos Spanos ${ }^{1}$, Biljana Glišić ${ }^{2}$, Milan Petakov, \\ David Rousso $^{1}$, Anargyros Kourtis ${ }^{1}$, Jelica Bjekić ${ }^{3}$ and Nataša Milić ${ }^{4}$ \\ Institute of Endocrinology, Diabetes and Metabolic Diseases, Clinical Center of Serbia, Dr Subotića 13, 11000 Belgrade, Serbia, ${ }^{1}$ Division of Endocrinology \\ and Human Reproduction, 2nd Department of Obstetrics and Gynecology, Aristotle University of Thessaloniki, Konstantinoupoleos 49, 54642 \\ Thessaloniki, Greece, ${ }^{2}$ Institute of Medical Biochemistry, Clinical Center of Serbia, Višegradska 26, 11000 Belgrade, Serbia, ${ }^{3}$ Department of \\ Endocrinology, CHC Bežanijska kosa, Autoput bb, 11000 Belgrade, Serbia and ${ }^{4}$ Institute of Medical Statistics, School of Medicine, Dr subotića 15, 11000 \\ Belgrade, Serbia \\ (Correspondence should be addressed to D Macut; Email: macut@EUnet.yu)
}

\begin{abstract}
Objective: Women with polycystic ovary syndrome (PCOS) are assumed to be at increased risk for cardiovascular diseases. This study examined the variations in oxidised low-density lipoprotein (OxLDL) concentration in relation to insulin levels in young women with PCOS.

Design: Cross-sectional clinical study in tertiary cares research hospitals. A total of 179 women with PCOS (79 overweight) and 56 age- and body mass index-matched controls were examined.

Methods: Blood samples were collected in follicular phase of the cycle for the basal glucose, total-, highdensity lipoprotein-cholesterol (HDL-C) and LDL-cholesterol, OxLDL, triglycerides, apolipoprotein-A1 (Apo-A1) and B (Apo-B), lipoprotein (a), insulin, testosterone and sex hormone-binding globulin (SHBG). Homeostatic model index (HOMA) and free androgen index (FAI) were determined. Results: Overweight and normal weight women with PCOS had higher concentrations of OxLDL than their control counterparts $(P=0.007$ and 0.003 respectively). Both the basal insulin $(P=0.003)$ and HOMA values $(P<0.001)$ were significantly higher in overweight than normal weight patients. Testosterone and FAI were higher in patients than in the respective controls $(P<0.001)$. The only independent predictor of increased OxLDL concentration in normal weight patients was Apo-B-to-Apo-A1 ratio $(P<0.001$, odds ratio (OR) 6.1; 95\% confidence interval (CI) 2.3-16.4), while in obese PCOS, it was total cholesterolto-high-density lipoprotein cholesterol ratio $(P<0.001$, OR 2.8 ; 95\% CI 1.6-4.9).

Conclusion: Young normal weight and overweight PCOS women have similarly increased OxLDL levels. Our results may indicate the presence of primary alteration in lipid metabolism in patients with PCOS. To answer the question whether the alteration in LDL particle size can by itself pose a higher cardiovascular risk, a careful follow-up of these women is needed.
\end{abstract}

European Journal of Endocrinology 155 131-136

\section{Introduction}

Polycystic ovary syndrome (PCOS) is a common reproductive endocrine disorder that is characterised by hyperandrogenism and chronic anovulation, affecting up to $10 \%$ of reproductive-aged women $(1,2)$. Today, PCOS is considered to be a metabolic disorder closely related to obesity, insulin resistance, hyperinsulinaemia, and atherogenic lipid profile (3-6).

Although the incidence of cardiovascular disease in PCOS is still a controversial issue (7-9), considerable attention in the past decade has been paid to this problem. Dyslipidaemia is the most common metabolic abnormality in PCOS, although the type and the extent of findings have not been defined. According to the National Cholesterol Educational Programme guidelines, prevalence of an abnormal lipid level is either borderline normal or high, approaching almost 70\% (5). In vast majority of patients with PCOS, using current cutoffs established by this programme, mean lipid values fall within the normal limits (3, 5, 6, 9). Additionally, insulin resistance has been associated with decreased levels of high-density lipoprotein cholesterol (HDL-C), increased levels of low-density lipoprotein cholesterol (LDL-C) and triglycerides $(5,8)$. Moreover, in these patients, the concentration of small dense LDL particles has been associated with three- to sevenfold increased relative risk of coronary artery disease (CAD) (10). Similarly, clinical signs of hyperandrogenism as hirsutism and acne appear to be associated with angiographic evidence of CAD in women with PCOS (11).

Recently, elevated levels of oxidised low-density lipoprotein (OxLDL) have been detected in patients with CAD (12-15), establishing the role of OxLDL in the 
initiation and progression of atherosclerosis. In these studies, clinical predictors of elevated OxLDL levels were female sex, family history of premature cardiovascular disease, increased body mass index (BMI) and percent of body fat, as well as exercise less than four times per week (12). Furthermore, circulating levels of OxLDL were predictive for $\mathrm{CAD}$ with relatively high sensitivity and specificity (15).

Whether patients with PCOS have elevated levels of OxLDL is unknown. The aim of this study is to determine the values of OxLDL in these women and probably to disclose its association with insulin resistance as another major factor involved in the process of atherosclerosis.

\section{Materials and methods}

\section{Materials}

A total of 179 women with PCOS were examined. All patients came from the metropolitan areas of Belgrade and Thessaloniki, were Caucasians and had similar socio-cultural background. Subjects were recruited over a 12-month period from the outpatient clinics of every institution that conducted the study, where the patients were referred from different sources including selfreferral, obstetrics-gynaecology clinics and primary care physicians for investigation of oligo- or amenorrhoea, fertility problem, hirsutism, or acne.

PCOS was defined according to the revised 2003 Rotterdam Consensus conference on diagnostic criteria for PCOS (16). Besides moderate oligo/amenorrhoea, our patients had elevated serum testosterone concentrations and polycystic appearance of their ovaries.

Exclusion criteria for all the subjects included: inability to comply with study requirements, the presence of impaired fasting glucose (fasting venous glucose $\geq 6.0 \mathrm{mmol} / \mathrm{l}$ ), pregnancy, hypothyroidism, non-classical 21-hydroxylase deficiency, hyperprolactinaemia, Cushing's disease and androgen-secreting tumours excluded by appropriate tests, history of drug or alcohol abuse and a history of breast or uterine cancer, or cardiovascular disorder. No subjects had received any oral contraceptives, glucocorticoids, antiandrogens, ovulation induction agents, anti-diabetic and anti-obesity drugs, or other hormonal drugs for at least 3 months before the study.

In all examined subjects, BMI was calculated and waist-to-hip ratio (WHR) determined. The patients and controls were categorised with respect to BMI values to those having normal weight (BMI $\leq 25 \mathrm{~kg} / \mathrm{m}^{2}$ ) and those that were overweight (BMI $>25 \mathrm{~kg} / \mathrm{m}^{2}$ ).

An age- and BMI-matched control group consisted of 56 women with no signs of hyperandrogenism, either clinical or biochemical, prior to the study. The normal ovulatory state was confirmed by transvaginal ultrasonography and plasma progesterone levels during the luteal phase of the cycle.
This study was approved by the Institutional Ethical Committees and written consent was obtained from all subjects.

\section{Biochemical and hormonal testing}

Women with PCOS were examined during early follicular phase (within the first 6 days after the onset of menstruation in the case of mild oligomenorrhoea) or at random in case of severe oligo- or amenorrhoea. Controls were investigated in early follicular phase. In all PCOS patients and controls, blood samples were collected in the morning subsequent to overnight fast. Basal serum levels of total cholesterol (TC), HDL-C, LDL-C, OxLDL, triglycerides, apolipoprotein-A1 (Apo-A1), apolipoprotein-B (Apo-B), lipoprotein(a) (Lp(a)), glucose, insulin, testosterone and sex hormone-binding globulin (SHBG) were measured, and TC-to-HDL-C ratio (TC/HDL-C), LDL-C-to-HDL-C ratio (LDL-C/HDL-C), Apo-B-to-Apo-A1 ratio (Apo-B/Apo-A1) and triglyceride-to-HDL-C ratio (triglyceride/HDL-C) were determined in all PCOS patients and respective controls. Samples for the determination of OxLDL, insulin, testosterone and SHBG were stored at $-20^{\circ} \mathrm{C}$ until the analysis.

\section{Assays}

TC (mmol/l) and triglycerides (mmol/l) were measured by standard enzymatic methods (cholesterol: cholesterol oxidase, Randox, Belfast, UK; triglycerides: glycerol-3phosphat oxidase, Randox, UK). HDL-C (mmol/l) was measured by direct method (Randox, UK). LDL-C $(\mathrm{mmol} / \mathrm{l})$ was determined by Friedewald formula (17). OxLDL (U/l) was measured by commercially available solid phase two-site enzyme immunoassay, sandwich ELISA (Mercodia AB, Uppsala, Sweden). The intra- and interassay coefficients of variation $(\mathrm{CV})$ were 4.0 and 6.2\% respectively. Apo-A1 (g/l), Apo-B (g/l) and Lp(a) $(\mathrm{g} / \mathrm{l})$ were determined by nephelometric method (Behring, Marburg, Germany). Plasma glucose (mmol/l) was determined by glucose oxidase method (Randox, UK), using the auto-analyser (Beckman, Brea, CA, USA). Plasma insulin (mU/l) levels were determined using two-site enzyme immunoassay, sandwich ELISA (Mercodia AB, Uppsala, Sweden). The intra- and interassay CV were 2.6 and $4.0 \%$ respectively. Serum testosterone (nmol/l) was measured by RIA (TESTOCT2, CIS bio international, Gif-Sur-Yvette Cedex, France). The intra- and interassay $C V$ was 4.5 and $5.1 \%$ respectively. SHBG $(\mathrm{nmol} / \mathrm{l})$ was measured by RIA (SHBG-RIACT, CIS bio international, Gif-Sur-Yvette Cedex, France). The intra- and interassay CV was 3.9 and $4.7 \%$ respectively. Free androgen index (FAI) was calculated from total testosterone and SHBG, using the formula $\mathrm{FAI}=(100 \times \mathrm{T}) / \mathrm{SHBG}$, with both testosterone and SHBG expressed in nmol/l (18). FAI $>8$ was considered significant. 


\section{Measures of insulin sensitivity}

Insulin sensitivity was calculated by homeostatic model index (HOMA). HOMA was calculated using the formula [fasting insulin $(\mathrm{mU} / \mathrm{l}) \times$ fasting glucose $(\mathrm{mmol} / \mathrm{l})] / 22.5$ (19).

\section{Data analysis}

Results were presented as the mean \pm s.D. Differences between groups were assessed by using ANOVA with Tukey's honestly significant difference test for pair-wise multiple comparisons or non-parametric Mann-Whitney $\mathrm{U}$ test, with Bonferroni correction for $P$-values for multiple comparisons, as appropriate. Associations between different variables were determined by using Pearson's Correlation Coefficient. Stepwise multivariate logistic regression analysis was used to identify independent predictors of elevated OxLDL levels. Only variables that were significant in univariate analyses entered the equation. $P<0.05$ was considered significant.

\section{Results}

\section{Clinical characteristics of patients}

Clinical and biochemical characteristics of patients and control subjects are presented in Table 1 . There was no difference in age, TC and LDL-C, Lp(a) and basal glucose concentrations between control subjects and PCOS patients.

Other parameters of lipid metabolism were significantly different in women with PCOS than in healthy women. Concentrations of HDL-C were lower, while concentration of triglycerides was higher in overweight patients, but this was not the case in control subjects. In the controls, Apo-A1 was lower in overweight than in normal weight subjects, whereas in patient's group, the concentration of Apo-B was higher in overweight than in normal weight subjects. Thus, proatherogenic index Apo-B/Apo-A1 as well as TC/HDL-C and LDL-C/HDL-C ratios were significantly higher in overweight PCOS patients than in normal weight controls. In addition, overweight patients had significantly higher values of TC/HDL-C, LDL-C/HDL-C and triglyceride/HDL-C ratios in comparison to normal weight patients with PCOS. Women with PCOS had higher concentrations of OxLDL than control subjects.

Overweight women with PCOS were more insulin resistant than normal weight patients. They had higher basal insulin levels and HOMA values (Table 1). There was no difference between insulin resistance of normal weight and overweight control subjects. Normal weight control subjects were more insulin sensitive than overweight PCOS patients. Although basal testosterone concentrations between normal weight and overweight PCOS patients were similar (Table 1), significantly

Table 1 Clinical and biochemical features, and indices of insulin sensitivity in groups of PCOS patients and controls in relation to body mass index (BMI).

\begin{tabular}{|c|c|c|c|c|c|}
\hline & \multicolumn{2}{|c|}{ PCOS patients } & \multirow{2}{*}{$\begin{array}{l}\text { Controls } \\
\text { Overweight } \\
(N=20)\end{array}$} & \multirow[b]{2}{*}{$\begin{array}{l}\text { Normal weight } \\
\quad(N=36)\end{array}$} & \multirow[b]{2}{*}{$P$ value } \\
\hline & $\begin{array}{c}\text { Overweight } \\
(N=79)\end{array}$ & $\begin{array}{l}\text { Normal weight } \\
\quad(N=100)\end{array}$ & & & \\
\hline Age & $24.9 \pm 5.9$ & $23.8 \pm 4.0$ & $25.7 \pm 5.9$ & $23.6 \pm 4.4$ & 0.121 \\
\hline $\mathrm{BMl}$ & $30.2+4.4^{\mathrm{a}}$ & $21.4+2.02$ & $28.9+3.5^{\mathrm{e}}$ & $20.49+1.9$ & $<0.001$ \\
\hline WHR & $0.83 \pm 0.07^{a}$ & $0.76 \pm 0.04$ & $0.81 \pm 0.07^{\mathrm{e}}$ & $0.75 \pm 0.03$ & $<0.001$ \\
\hline TC & $4.8 \pm 0.9$ & $4.6 \pm 0.9$ & $4.6 \pm 0.6$ & $4.4 \pm 0.8$ & 0.141 \\
\hline LDL-C & $2.9 \pm 0.9$ & $2.7 \pm 0.9$ & $2.9 \pm 0.6$ & $2.5 \pm 0.6$ & 0.139 \\
\hline HDL-C & $1.2 \pm 0.3^{a}$ & $1.4 \pm 0.3$ & $1.3 \pm 0.3$ & $1.5 \pm 0.3$ & $<0.001$ \\
\hline $\mathrm{TC} / \mathrm{HDL}-\mathrm{C}$ & $4.09+1.4^{\mathrm{a}, \mathrm{c}}$ & $3.2+0.8$ & $3.8+1.4$ & $3.03+0.6$ & $<0.001$ \\
\hline LDL-C/HDL-C & $2.5 \pm 1.1^{\mathrm{a}, \mathrm{f}}$ & $1.9 \pm 0.8$ & $2.2 \pm 0.7$ & $1.8 \pm 0.5$ & $<0.001$ \\
\hline Triglycerides & $1.4 \pm 0.8^{\mathrm{a}}$ & $0.9 \pm 0.6$ & $1.2 \pm 1.3$ & $0.8 \pm 0.2$ & $<0.001$ \\
\hline Triglyceride/HDL-C & $1.3 \pm 0.9^{a}$ & $0.7 \pm 0.5$ & $1.4 \pm 2.8^{\mathrm{m}}$ & $0.7 \pm 0.5$ & $<0.001$ \\
\hline Apo-A1 & $1.6 \pm 0.3$ & $1.6 \pm 0.3$ & $1.5 \pm 0.2^{1}$ & $1.7 \pm 0.3$ & 0.014 \\
\hline Apo-B & $0.9 \pm 0.2^{k}$ & $0.8 \pm 0.2$ & $0.8 \pm 0.2$ & $0.8 \pm 0.1$ & 0.007 \\
\hline Apo-B/Apo-A1 & $0.6 \pm 0.2^{n}$ & $0.5 \pm 0.3$ & $0.6 \pm 0.1$ & $0.5 \pm 0.1$ & 0.035 \\
\hline Lp(a) & $0.2 \pm 0.2$ & $0.2 \pm 0.2$ & $0.2 \pm 0.2$ & $0.1 \pm 0.1$ & 0.475 \\
\hline OxLDL & $65.4 \pm 26.1^{i}$ & $61.5 \pm 26.8^{h}$ & $44.8 \pm 7.8$ & $44.3 \pm 8.5$ & $<0.001$ \\
\hline Basal glucose & $4.9 \pm 0.7$ & $4.8 \pm 0.6$ & $4.8 \pm 1.0$ & $4.5 \pm 0.7$ & 0.126 \\
\hline Basal insulin & $15.6 \pm 10.2^{\mathrm{c}, \mathrm{g}}$ & $10.8 \pm 6.3$ & $10.6 \pm 5.8$ & $7.8 \pm 3.2$ & $<0.001$ \\
\hline HOMA & $3.6 \pm 2.6^{\mathrm{a}, \mathrm{c}}$ & $2.4 \pm 1.3$ & $2.4 \pm 1.5$ & $1.6 \pm 0.7$ & $<0.001$ \\
\hline Testosterone & $3.3 \pm 1.2^{\mathrm{b}}$ & $3.1 \pm 1.0^{\mathrm{d}}$ & $1.6 \pm 0.4$ & $1.4 \pm 0.7$ & $<0.001$ \\
\hline SHBG & $27.5 \pm 11.6^{\mathrm{b}, \mathrm{j}}$ & $35.1 \pm 13.4^{d}$ & $45.1 \pm 14.4$ & $58.2 \pm 22.4$ & $<0.001$ \\
\hline FAl & $16.1 \pm 12.2^{a, b}$ & $10.2 \pm 5.0^{\mathrm{d}}$ & $4.1 \pm 2.2$ & $3.0 \pm 2.4$ & $<0.001$ \\
\hline
\end{tabular}

${ }^{*} P$ values for comparison of all four groups and for two-way comparison of patients and controls that were significant in the initial four-way comparisons. ${ }^{a} P<0.001$ overweight PCOS vs normal weight PCOS, ${ }^{\mathrm{b}} P<0.001$ overweight PCOS vs overweight controls, $P<0.001$ non-obese PCOS vs normal weight controls, ${ }^{\mathrm{e}} P<0.001$ overweight controls vs normal weight controls, ${ }^{\mathrm{f}} P=0.001$ overweight $\mathrm{PCOS}$ vs normal weight controls, ${ }^{\mathrm{g}} P=0.003$ overweight $\mathrm{PCOS}$ vs normal weight PCOS, ${ }^{\mathrm{h}} P=0.003$ non-obese PCOS vs normal weight controls, ${ }^{\mathrm{i}} P=0.007$ overweight $\mathrm{PCOS}$ vs overweight controls, ${ }^{\mathrm{j}} P=0.01$ overweight PCOS vs normal weight PCOS, ${ }^{\mathrm{k}} P=0.013$ overweight $\mathrm{PCOS}$ vs normal weight $\mathrm{PCOS},{ }^{\mathrm{I}} P=0.021$ overweight controls vs normal weight controls, $\mathrm{m} P=0.034$ overweight controls vs normal weight controls, ${ }^{n} P=0.04$ overweight PCOS vs normal weight controls. 
higher values of FAI were found in overweight patients. There was no difference in testosterone levels and FAI within control subjects. Significant differences between these parameters in control subjects and PCOS patients was found in our study.

\section{Correlations in patients with PCOS}

In all patients, BMI correlated positively with FAI $(r=0.38 ; P<0.001)$, basal insulin $(r=0.41 ; P<0.001)$ , HOMA $(r=0.42 ; P<0.001)$, testosterone concentration $(r=0.16 ; P=0.016)$, TC/HDL-C $(r=0.41 ; P<0.001)$, LDL-C/HDL-C $(r=0.33 ; P<0.001)$, and triglyceride/HDL-C $(r=0.33 ; P<0.001)$. OxLDL was in positive correlation with age $(r=0.22 ; P=0.006)$, BMI $(r=0.14$; $P=0.036)$, insulin $(r=0.33 ; P<0.001)$ and testosterone $(r=0.27 ; P<0.001)$ concentrations, HOMA $(r=0.31$; $P<0.001)$ and FAI $(r=0.29 ; P<0.001)$ indices. Indexes of cardiovascular risk, TC/HDL-C and Apo-B/Apo-A1 were in positive correlation with testosterone $(r=0.17$; $P=0.021$ and $r=0.26 ; P<0.001)$ and FAI index $(r=0.18 ; P=0.026$ and $r=0.23 ; P=0.002)$, while LDL-C/HDL-C was in positive correlation with testosterone concentration $(r=0.19 ; P=0.012)$. Basal insulin levels and HOMA index were in positive correlation with testosterone concentration $(r=0.23 ; P=0.001$, for both $)$, FAI index $(r=0.39 ; P<0.001$ and $r=0.36 ; P<0.001)$, TC/HDL-C $(r=0.38 ; P<0.001$ and $r=0.4 ; P<0.001)$, LDL-C/HDL-C $\quad(r=0.34 ; \quad P<0.001$ and $r=0.36$; $P<0.001)$, Apo-B/Apo-A1 $(r=0.34 ; P<0.001$ and $r=0.32 ; P<0.001)$ and triglyceride/HDL-C $(r=0.34$; $P<0.001$, for both).

In overweight PCOS patients, our analysis found the correlation of WHR with basal insulin and HOMA index $(r=0.29 ; P=0.024)$, while in normal weight PCOS patients, it was in association with serum lipids: cholesterol $(r=0.41 ; P<0.001)$, LDL-C $(r=0.39$; $P<0.001)$, Apo-B $(r=0.35 ; \quad P=0.001)$, OxLDL $(r=0.27 ; P=0.009)$, and lipid ratios of TC/HDL-C and LDL-C/HDL-C ( $r=0.34 ; P=0.001$, for both).

Insulin was in correlation with TC/HDL-C $(r=0.5$; $P<0.001)$, LDL-C/HDL-C $(r=0.52 ; P<0.001)$ and Apo-B/Apo-A1 $(r=0.28 ; P=0.026)$ in overweight PCOS patients. Similarly, HOMA index was in association with these indices $(r=0.47 ; P<0.001, r=0.5$; $P<0.001$ and $r=0.29 ; P<0.024$ respectively). LDL-C/HDL-C correlated with testosterone concentration significantly in overweight PCOS patients $(r=0.25 ; P=0.026)$.

\section{Logistic regression analysis}

In both overweight and normal weight patients, similar variables were found to be statistically significant in univariate analyses. The only independent predictor of OxLDL in normal weight PCOS women, based on standard clinical criteria, was Apo-B/Apo-A1, while in overweight patients, the independent predictor of disease was TC/HDL-C (Table 2).

\section{Discussion}

This study revealed that overweight and normal weight women with PCOS had similarly increased concentrations of OxLDL in comparison to healthy women. Independent predictors of elevated OxLDL levels in both overweight and normal weight patients were only proatherogenic lipid indices.

Difference of FAI and HOMA index values between overweight and normal weight PCOS women was not translated into different plasma concentrations of OxLDL in these patients. Obesity and atherogenic lipid profile are often found in women with PCOS (20). The most consistent alterations in lipid metabolism associated with metabolic syndrome are elevated triglycerides and lower HDL-C concentrations with increase of the triglyceride/HDL-C ratio (7, 9, 20-22) as it was the case with overweight patients and control subjects in our study. However, similar increase of OxLDL levels in overweight and normal weight women with PCOS in this study supports the viewpoint that association of insulin-resistant syndrome and LDL particle size is not necessarily mediated by hyperinsulinaemia but via alterations in lipid metabolism itself (23).

It has been recently shown that young normal weight, non-dyslipidaemic and non-hypertensive women with PCOS have an early impairment of endothelial structure and function (24). Since, OxLDL was not measured in this study, these observations could be in line with our results, as young normal weight patients in our study had normal traditional lipid measures. The role of increased levels of small OxLDL particles in atherogenesis has been extensively studied only in patients with cardiovascular disease (13-15, 21, 25). These patients showed consistency in proving upregulation of OxLDL production and this is concordant with our results (21). It is likely that LDL atherogenicity increases simultaneously with reduction of LDL particle size (26). Our results are in line with findings of Dejager et al. (27), who showed that women with PCOS had reduced size of LDL-C particles in

Table 2 Variables independently associated with OxLDL*.

\begin{tabular}{lcc}
\hline Independent variables & $\begin{array}{c}\text { Odds ratio (95\% } \\
\text { Confidence Interval) }\end{array}$ & $\boldsymbol{P}$ value \\
\hline $\begin{array}{c}\text { Normal weight patients: } \\
\text { Apo-B/Apo-A1 }\end{array}$ & $6.1(2.3-16.4)$ & $<0.001$ \\
$\begin{array}{l}\text { Overweight patients: Total } \\
\text { cholesterol/HDL-C }\end{array}$ & $2.8(1.6-4.9)$ & $<0.001$ \\
\hline
\end{tabular}

*In univariate logistic regression analyses, the following variables were statistically significant: WHR, testosterone, FAI, SHBG, total cholesterol/HDL-C, LDL-C/HDL-C, triglyceride/HDL-C, basal insulin and HOMA. 
comparison with normal cycling women. The influence of increased OxLDL concentrations on early atherogenesis in women with PCOS has not been studied. Thus, careful follow up of these women is needed to prove causality between the increased susceptibility to atherosclerosis and reduced diameter of LDL particles (23).

It is likely that obesity may cause aggravation of the existing functional hyperandrogenism and alterations in traditional lipid measures by increasing the insulin resistance (28), as it has been demonstrated in our study as well. However, complete normalisation of testosterone levels by long-acting GnRH agonist does not influence at all the insulin resistance in women with PCOS, consequently demonstrating that hyperandrogenaemia does not play a role in their insulin resistance (29). Besides impact on clinical characteristics of the syndrome, the existing insulin resistance of PCOS may also be involved in other metabolic disorders, since higher prevalence of type 2 diabetes within families of obese (54.8\%) and non-obese (24.2\%) women with PCOS has been demonstrated (30).

In conclusion, our results provide the evidence about the presence of primary alteration in lipid metabolism in young patients with PCOS. Whether the elevation of OxLDL in these young women is associated with silent phase of atherosclerosis remains to be elucidated.

\section{Acknowledgement}

This research project was supported by grant of the Ministry of Science of Serbia (143003).

\section{References}

1 Franks S. Polycystic ovary syndrome. New England Journal of Medicine 1995333 853-861.

2 Knochenhauer ES, Key TJ, Kahsar-Miller M, Waggoner W, Boots LR \& Azziz R. Prevalence of the polycystic ovary syndrome in unselected black and white women of the southeastern United States: a prospective study. Journal of Clinical Endocrinology and Metabolism $1998 \mathbf{8 3}$ 3078-3082.

3 Wild RA, Painter PC, Coulson PB, Carruth KB \& Ranney GB. Lipoprotein lipid concentrations and cardiovascular risk in women with polycystic ovary syndrome. Journal of Clinical Endocrinology and Metabolism $1985 \mathbf{6 1}$ 946-951.

4 Conway GS, Agrawal R, Betteride DJ \& Jacobs HS. Risk factors for coronary artery disease in lean and obese women with the polycystic ovary syndrome. Clinical Endocrinology 199237 127-134.

5 Legro RS, Kunselman AR \& Dunaif A. Prevalence and predictors of dyslipidemia in women with polycystic ovary syndrome. American Journal of Medicine 2001111 607-613.

6 Apridonidze T, Essah PA, Iuorno MJ \& Nestler JE. Prevalence and characteristics of the metabolic syndrome in women with polycystic ovary syndrome. Journal of Clinical Endocrinology and Metabolism 200490 1929-1935.

7 Dahlgren E, Janson PO, Johansson S, Lapidus L \& Oden A. Polycystic ovary syndrome and risk for myocardial infarction.
Evaluated from a risk factor model based on a prospective population study of women. Acta Obstetrica et Gynecologica Scandinavica 199271 599-604.

8 Talbott E, Clerici A, Berga SL, Kuller L, Guzick D, Detre K, Daniels T \& Engberg RA. Adverse lipid and coronary heart disease risk profiles in young women with polycystic ovary syndrome: results of a case-control study. Journal of Clinical Epidemiology $1998 \mathbf{5 1}$ 415-422.

9 Wild S, Pierpoint T, McKeigue PM \& Jacobs H. Cardiovascular disease in women with polycystic ovary syndrome at long-term follow-up: a retrospective cohort study. Clinical Endocrinology 2000 52 595-600.

10 Austin MA, Breslow JL, Hennekens CH, Buring JE, Willett WC \& Krauss RM. Low-density lipoprotein subclass patterns and risk of myocardial infarction. Journal of the American Medical Association $19882601917-1921$.

11 Birdsall M, Farquhar C \& White H. Association between polycystic ovaries and extent of coronary artery disease in women having cardiac catheterization. Annals of Internal Medicine $199712632-35$.

12 Mosca L, Rubenfire M, Tarshis T, Tsai A \& Pearson T. Clinical predictors of oxidized low-density lipoprotein in patients with coronary artery disease. American Journal of Cardiology $1997 \mathbf{8 0}$ 825-830.

13 Holvoet P, Vanhaecke J, Janssens S, Van de Werf F \& Collen D. Oxidized LDL and malondialdehyde-modified LDL in patients with acute coronary syndromes and stable coronary artery disease. Circulation 199898 1487-1494.

14 Ehara S, Ueda M, Naruko T, Haze K, Itoh A, Otuska M, Komatsu R, Matsuo T, Itabe H, Takano T, Tsukamoto Y, Yoshiyama M, Takeuchi K, Yoshikawa J \& Becker AE. Elevated levels of oxidized low density lipoprotein show a positive relationship with the severity of acute coronary syndromes. Circulation $2001 \mathbf{1 0 3}$ 1955-1960.

15 Holvoet P, Mertens A, Verhamme P, Bogaerts K, Beyens G, Verhaeghe R, Collen D, Muls E \& Van de Werf F. Circulating oxidized LDL is a useful marker for identifying patients with coronary artery disease. Arteriosclerosis, Thrombosis and Vascular Biology $200121844-848$.

16 The Rotterdam ESHRE/ASRM-Sponsored PCOS Consensus Workshop Group. Revised 2003 consensus on diagnostic criteria and long-term health risk related to polycystic ovary syndrome. Fertility and Sterility 200481 19-25.

17 Friedewald WT, Levy RI \& Fredrickson DS. Estimation of the concentration of low-density lipoprotein cholesterol in plasma, without use of preparative ultracentrifuge. Clinical Chemistry 1972 18 499-502.

18 Mathur RS, Moody LO, Landgrebbe S \& Williamson HO. Plasma androgens and sex hormone binding globulin in the evaluation of hirsute patients. Fertility and Sterility 198135 29-37.

19 Matthews DR, Hosker JP, Rudenski AS, Naylor BA, Treacher DF \& Turner RC. Homeostasis model assessment: insulin resistance and beta-cell function from fasting plasma glucose and insulin concentrations in man. Diabetologia 1985 28 412-419.

20 Gambineri A, Pelusi C, Vicennati V, Pagotto U \& Pasquali R. Obesity and the polycystic ovary syndrome. International Journal of Obesity and Related Metabolic Disorders 200226 883-896.

21 Holvoet P, Kritchevsky SB, Tracy RP, Mertens A, Rubin SM, Butler J, Goodpaster B \& Harris TB. The metabolic syndrome, circulating oxidized LDL, and risk of myocardial infarction in well-functioning elderly people in the health, aging, and body composition cohort. Diabetes 200453 1068-1073.

$22 \mathrm{Gu}$ D, Reynolds K, Wu X, Chen J, Duan X, Reynolds RF, Whelton PK, He J \& InterASIA Collaborative Group. Prevalence of the metabolic syndrome and overweight among adults in China. Lancet 2005365 1398-1405.

23 Friedlander Y, Kidron M, Caslake M, Lamb T, McConnell M \& Bar-On H. Low density lipoprotein particle size and risk factors of insulin resistance syndrome. Atherosclerosis 2000148 141-149.

24 Orio F Jr, Palomba S, Cascella T, De Simone B, Di Base S, Russo T, Labella D, Zullo F, Lombardi G \& Colao A. Early impairment of 
endothelial structure and function in young normal-weight women with polycystic ovary syndrome. Journal of Clinical Endocrinology and Metabolism 200489 4588-4593.

25 Hulthe J \& Fagerberg B. Circulating oxidized LDL is associated with subclinical atherosclerosis development and inflammatory cytokines (AIR Study). Arteriosclerosis, Thrombosis and Vascular Biology 200222 1162-1167.

26 Tribble DL, Holl LG, Wood PD \& Krauss RM. Variations in the oxidative susceptibility among six low density lipoprotein subfractions of differing density and particle size. Atherosclerosis 1992 93 189-199.

27 Dejager S, Pichard C, Giral P, Bruckert E, Federspield MC, Beucler I \& Turpin G. Smaller LDL particle size in women with polycystic ovary syndrome compared to controls. Clinical Endocrinology 2001 54 455-462.
28 Wild RA. Obesity, lipids, cardiovascular disease risk and androgen excess. American Journal of Medicine 199598 (1A) 27S-32S.

29 Dunaif A, Green G, Futterweit W \& Dobrjansky A. Suppression of hyperandrogenism does not improve peripheral or hepatic insulin resistance in the polycystic ovary syndrome. Journal of Clinical Endocrinology and Metabolism 1990 70 699-704.

30 Fox R. Prevalence of a positive family history of type 2 diabetes in women with polycystic ovarian disease. Gynecological Endocrinology 199913 390-393.

Received 8 January 2006

Accepted 28 April 2006 